\title{
THE RADIOACTIVE ION BEAM FACILITY PROJECT FOR THE LEGNARO LABORATORIES
}

\author{
L.B. Tecchio on behalf of the SPES Study Group \\ Laboratori Nazionali di Legnaro, Via Romea 4, 35020 Legnaro (PD), Italy
}

\begin{abstract}
In the frame work of the Italian participation to the project of a high intensity proton facility for the energy amplifier and nuclear waste transmutations, the LNL has been proposed a project for the construction of a second generation facility for the production of radioactive ion beams (RIBs) by using the ISOL method. The final goal consists in the production of neutron rich RIBs with masses ranging from 80 to 160 by using primary beams of protons/ deuterons with energy of $100 \mathrm{MeV} / \mathrm{u}$ and 100 $\mathrm{kW}$ power. This project is proposed to be developed in about 10 years from now and intermediate milestones and experiments are foreseen and under consideration for the next INFN five year plan (1999-2003). In such period of time is proposed the construction of a deuteron accelerator of $20 \mathrm{MeV}$ energy and $5 \mathrm{~mA}$ current, consisting of a RFQ $(5 \mathrm{MeV})$ and a linac $(20 \mathrm{MeV})$, and of a neutron area dedicated to the RIBs production and other applications. Besides the RIBs production, neutron beams for the BNCT applications and neutron physics are also planned.
\end{abstract}

\section{METHODS OF PRODUCTION}

Two methods can be used to produce RIBs and are the Projectile Fragmentation (PF) and the post-acceleration of isotopes produced by spallation, fragmentation, or fission reactions with high-intensity proton/light-ion beams in thick targets (ISOL method). The ISOL method takes a complementary approach to the production of RIBs. In the ISOL scheme a primary accelerator (or a nuclear reactor) yields a production beam of charged particles (or neutrons), which is sent on a thick, hot target. The radioactive species thereby produced are transported by a transfer tube to an ion source, then mass separated and breeding to higher charge state; the resulting ions are separated by an isotope/isobar separator, post-accelerated and sent into the experimental area. The comparison and choice between the PF and ISOL methods is strongly dependent on the physics to investigate. One of the relevant parameters for a comparison of the ISOL and the $\mathrm{PF}$ production methods is the RIBs production luminosity, which is independent of cross-section. The maximum production luminosity expected for an ISOL facility is three or four order of magnitude higher than in the PF figure. This because the luminosity can be achieved through the combination of a thick production target and a very intense primary beam. The proposed researches which are done at near-Coulomb barrier energies and below, involves studies of nuclear structure, low-energy nuclear reactions, astrophysics, and materials sciences applications and are best served by intense, highquality, low-energy RIBs provided by the ISOL approach. This because the post-accelerator, optimized for high quality beams, provide the easy energy variability, highenergy precision and small emittances as demanded by the experiments. The main drawback to the ISOL method is that the diffusion/desorption from the production target and ionization of radioactive fragments are strongly element-dependent and slower than in the PF method. However, this feature can be also an advantage of the ISOL method because it give $\mathrm{Z}$ selectivity and, consequently, enhance beam purity.

\section{THE FACILITY CONCEPT}

We propose a two-accelerator ISOL-type facility to provide intense neutron-rich radioactive ion beams of highest quality, in the range of masses between 80 and 160. The proposed production mechanism is the fission induced by fast neutrons in fissile material targets. The fission mechanism has the advantage that very neutron rich radioactive beams can be produced. The conceptual design is based on a high intensity proton/deuteron linac as driver and on the availability of the heavy-ion accelerator ALPI as post accelerator [1]. The main idea is to use the primary beams to produce, by a converting target, an intense flux $\left(\sim 3 \times 10^{14} \mathrm{~cm}^{-2} \mathrm{~s}^{-1}\right)$ of neutrons. The use of a converting target partially solves the problems concerning the beam power dissipation and radiation damage in the radioisotope production target/ion source system. The purely nuclear mechanism of energy loss and the long collision length of neutrons allow rather large target thickness to be used for more effective isotope production. The primary accelerator is a sequence of RFQDTL linac which can deliver proton/deuteron beams in the energy range of $10-100 \mathrm{MeV} / \mathrm{u}$ at a beam power of 100 $\mathrm{kW}$. The RFQ accelerates the beam up to $5 \mathrm{MeV}$ while further acceleration up to $100 \mathrm{MeV} / \mathrm{u}$ is accomplished by the DTL linac. The facility is planned to be located below ground level to assist in the prompt neutron radiation shielding. The beams delivered by the driver will produce radionuclides by irradiating targets in a well-shielded dedicated area. The radionuclides will be extracted at $20 \mathrm{kV}$ from the target and ionized to the 1+ charge state, charge breeded to obtain a mass over charge ratio of about 10 , mass separated, and then sent either directly to an experimental area for research with ion traps, or to the secondary-beam accelerator which will be housed in the existing ALPI building. To fill the ALPI linac with these 
very low energy beam requires a new accelerating stage (pre-accelerator) up to about $1 \mathrm{MeV} / \mathrm{u}$. The pre-accelerator consists of three low-frequency RFQs similar to the ones of the PIAVE injector [2]. These low-energy secondary beams can be delivered to the experimental area, located in the ALPI building, for low-energy experiments (astrophysics). The further acceleration up to about 5 $\mathrm{MeV} / \mathrm{u}\left({ }^{132} \mathrm{Sn}^{13+}\right)$ is accomplished by the ALPI linac and the radioactive beams will be delivered to the existing experimental areas. The capability of the ALPI linac to accelerate stable beams will remain independent of this program, both during construction and operation with radioactive beams.

\section{SECONDARY BEAM INTENSITIES}

Extensive calculations of production rates of radioisotopes have been performed using Montecarlo codes LAHET [3] and FLUKA [4]. The goal of such a calculations is to provide realistic and accurate estimates of the production yields for secondary ion beams to guide the detailed accelerator design and providing input for the physics program. Since LAHET code does not transport neutrons with energy below $20 \mathrm{MeV}$, simulations in voluminous thick targets have been performed with the FLUKA code (version '98). In particular, the fission process is computed using ENDF/B-6, JEF2.2 and JENDL3 nuclear data libraries. The most efficient production of neutronrich isotopes is achieved via neutron induced fission of fissile materials (i.e. uranium and thorium). Neutrons are produced by stopping the primary proton/deuteron beam in a suitable target (stopping target). The stopping target must be chosen according to the primary beam in order to emit secondary neutrons in a forward narrow cone. It is understood that such a two-step device produces its best results when the secondary neutrons production is high. This could be accomplished, for instance, taking advantage of the deuteron stripping in reactions with light elements. The predicted fission fragments spectrum for high energy neutrons has been calculated with the FLUKA code and shown in Fig.1.

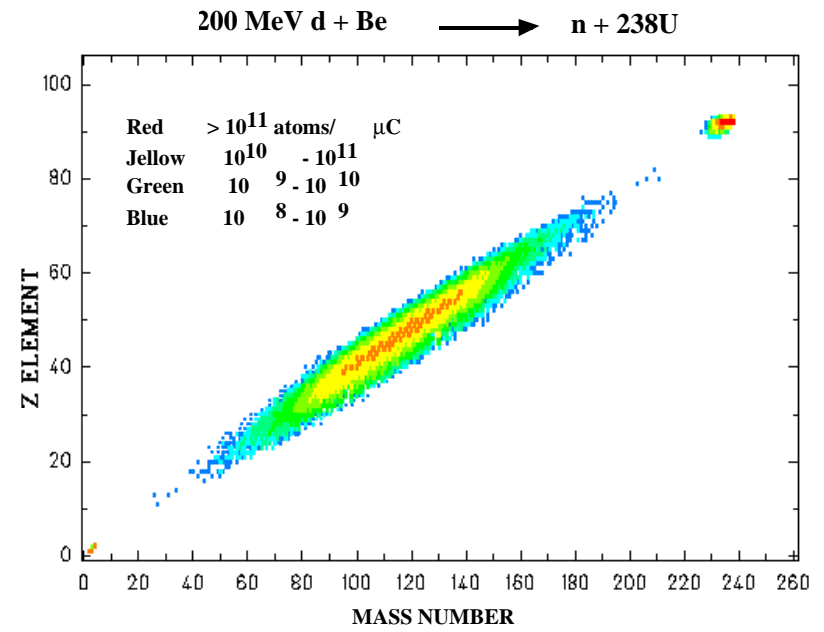

Figure 1: Predicted fission fragments spectrum.

\section{THE NEUTRON FACILITY SPES}

As an intermediate milestone of the full project the Legnaro Laboratories have been proposed a neutron facility (SPES) to be constructed in the next five years. It will result in a medium intensity neutron facility addressed to the national community and dedicated to the RIBs production as well as to the BNCT applications and to the neutron physics. Because its modularity the primary accelerator is planned to be constructed, at the beginning, in a minimal version and further implemented to accelerate beams up to $100 \mathrm{MeV} / \mathrm{u}$. The primary accelerator consist in the sequence of RFQ-DTL linac limited in energy $(10 \mathrm{MeV} / \mathrm{u})$. The beam power to be considered is ranging between 10 and $100 \mathrm{~kW}$. For the remaining part the facility is conceptually planned as described above, thus the secondary beams can be delivered for experiments at low-energy (traps, astrophysics,...) or pre-accelerated and injected in the ALPI linac for a further acceleration to higher energies. The limited energy of the primary accelerator will imply a lower neutron production rate; in any case the estimated neutron yield at the target level is $2 \times 10^{14} \mathrm{n} / \mathrm{s}(100 \mathrm{~kW})$, high enough to satisfy the demand for an advanced RIBs facility.

\section{NEUTRON PRODUCTION RATES AND BEAM INTENSITIES}

High energy neutron sources based on high current continuous wave (CW) deuteron or proton accelerators and thick targets of light nuclei provide the most suitable intensities for the aims of the project. The experimental data on deuteron and proton induced neutron source reactions were reviewed by Lone [5] and Barschall [6]. At deuteron energies above a few $\mathrm{MeV}$, the $\mathrm{d}+\mathrm{Be}$ and $\mathrm{d}+\mathrm{Li}$ reactions have the highest cross sections for production of neutrons in the forward direction. The high energy neutrons measured from thick beryllium and lithium targets exhibit spectral shapes and angular distributions which are characteristic of the deuteron stripping process. At a comparable projectile energy the intensity and the average energy of neutrons at $0^{0}$ from a thick beryllium target are highest for the deuterons. The dose rates from the $d+B e$ reaction on thick targets are higher than those from the $\mathrm{p}+\mathrm{Be}$ reaction at the same projectile energy. Also, the angular distributions of neutrons from the deuteron reactions are narrowed than those from the proton reactions at the same projectile energy. The intensity of the neutrons emitted in the forward direction increases rapidly with the deuteron bombarding energy. Independently from the stopping target, the deuterons seems the most appropriate projectiles for the production of neutrons. Stopping targets as beryllium and lithium both present characteristics which are suitable for the task. For some application uranium stopping target is suitable too, even if it shows different angular and energetic distributions. In this contest, for the intermediate milestone of the project, the most suitable driver may 
consists of a deuteron accelerator of $20 \mathrm{MeV}$ energy and power up to $100 \mathrm{~kW}$. With minimal changes to the RFQ designed for the high intensity proton driver the same device allows to accelerate also deuterons. The RFQ is followed by a short section of the DTL linac. The expected yield of neutrons with energy above $1 \mathrm{MeV}$ emitted in an angle between $0^{0}$ and $10^{0}$ is $2 \times 10^{14}(\mathrm{n} / \mathrm{s})$, for $100 \mathrm{~kW}$ power in the stopping beryllium target. The expected beam intensities for a production deuteron beam of $20 \mathrm{MeV}$ and $100 \mathrm{~kW}$ power are shown in Table 1. The stopping target is beryllium and the production target is uranium carbide with a thickness of $100 \mathrm{gcm}^{-2}$.

Table 1: Expected beam intensities. The overall efficiency values are taken from Ref. [7]. * Value taken from Ref. [8].

\begin{tabular}{|c|c|c|c|}
\hline Isotope & $\begin{array}{c}\text { Prod.Rate } \\
\text { 20 MeV } \\
{[\text { At./p } \mu \text { C] }]}\end{array}$ & $\begin{array}{c}\text { Overall } \\
\text { Efficiency } \\
{[\%]}\end{array}$ & $\begin{array}{c}\text { Beam Int. } \\
\text { 20 MeV } \\
\text { [Atom s/s] }\end{array}$ \\
\hline $\mathrm{Zn}^{72}$ & $1.3 \times 10^{3}$ & 8 & $5.2 \times 10^{5}$ \\
$\mathrm{Zn}^{78}$ & $1.2 \times 10^{6}$ & 0.48 & $2.9 \times 10^{7}$ \\
$\mathrm{Zn}^{84}$ & $2.4 \times 10^{2}$ & - & - \\
$\mathrm{Kr}^{91}$ & $9.4 \times 10^{8}$ & 29 & $1.4 \times 10^{12}$ \\
$\mathrm{Kr}^{94}$ & $2.6 \times 10^{8}$ & 6.4 & $8.3 \times 10^{10}$ \\
$\mathrm{Rb}^{97}$ & $3.2 \times 10^{7}$ & 11 & $1.7 \times 10^{10}$ \\
$\mathrm{Cd}^{131}$ & $10^{2}$ & $0.25^{*}$ & $10^{3}$ \\
$\mathrm{Sn}^{132}$ & $6.7 \times 10^{8}$ & 2.4 & $8 \times 10^{10}$ \\
$\mathrm{Sn}^{138}$ & $8 \times 10^{2}$ & $0.37^{*}$ & $1.5 \times 10^{4}$ \\
$\mathrm{Xe}^{142}$ & $5.5 \times 10^{8}$ & 26 & $7 \times 10^{11}$ \\
$\mathrm{Xe}^{144}$ & $4.4 \times 10^{7}$ & 26 & $5.7 \times 10^{10}$ \\
$\mathrm{Cs}^{144}$ & $6.3 \times 10^{8}$ & 38 & $1.2 \times 10^{12}$ \\
\hline
\end{tabular}

\section{RESEARCH AND DEVELOPMENT}

During the development of the conceptual design for the RIBs facility, numerous areas in which additional research and development seemed appropriated have been identified. These areas are briefly described below, but not in any order of priority.

\subsection{Stopping target for neutron production}

It was mentioned above that a beryllium or lithium targetconverter serves as an efficient neutron source for isotope production by fission in the secondary production target. This solution will allow to solve the technological problems related to the power dissipation $(100 \mathrm{~kW})$ from the production target by decoupling the intense particle beam from the secondary target in terms of the energy absorption and consequent target heating. Beryllium and lithium are suitable for such an application, but both kind of targets present design problems that demand a carefully investigation.

\subsection{Target/ion source}

In order to obtain experimental informations on the neutron-rich nuclei produced by neutron-induced fission of uranium isotopes an experimental program has been approved by INFN and actually in progress at LNL. It foresees the construction of an isotope separator for online operation coupled to the $7 \mathrm{MV} \mathrm{CN}$ van de Graaff accelerator. The layout of the isotope separator is very similar to presently existing low-current separators and consists of a $60^{\circ}$ magnet with $1.3 \mathrm{~m}$ bending radius, a mass resolving power M/_M 800 and dispersion $\sim 1500 / \mathrm{M} \mathrm{mm}$. The idea is to produce neutrons by bombarding thick beryllium targets with deuterons accelerated up to an energy of $7 \mathrm{MeV}$ and current of $3 \mu \mathrm{A}$. The expected total neutron yield at $7 \mathrm{MeV}$ deuteron energy is $5 \times 10^{9}$ neutrons $/ \mathrm{sr} / \mu \mathrm{A}$ at $0^{0}$, with an average energy $\left\langle\mathrm{E}_{\mathrm{n}}\right\rangle=3.2 \mathrm{MeV}$. The on-line test stand for study the production yields, the release efficiencies, the delay times in the production targets and for testing the ion source efficiencies and emittances. A high-temperature integrated target-ion-source system has been designed for the on-line mass separator. This system includes, other than the target, a 1+ charge state surface ionization source operating at $20 \mathrm{kV}$. The targets are composed of uranium carbide (typical thickness $100 \mathrm{~g} \mathrm{~cm}^{-2}$ ). In order to have a rapid diffusion and a good selectivity of the fission products the target-ion-source system can be operated up to $2500{ }^{0} \mathrm{C}$. The expected production rates at the target level for several different isotopes is estimate ranging in between $10^{4}-10^{6}$ atoms/s/grU.

\subsection{Production of multiple charged ions}

To accelerate radioactive beams efficiently, ions charge greater than +1 may be advantageous. Efficient production of such ions would also reduce the cost of the acceleration stages. A new ion source of the EBIS-type, the Trapped Ion Source (TIS) actually under development is capable, in principle, of producing very highly charged ions [9]. The main new feature of TIS, with respect to an EBIS, is the adding of radial ion confinement of the rf quadrupoles to the potential well of the electron beam space charge, which allow to select the ion type to be contained in the pseudo-potential well (as is done in a common mass spectrometers).

\section{REFERENCES}

[1]G. Fortuna et al., "Status of ALPI", LINAC'96, CERN 96-07.

[2]A. Lombardi et al.,"PIAVE, the Positive Ion Injector for ALPI", LINAC'96, CERN 96-07.

[3]R.E. Prael and M. Bozoian, LA-UR-88-3238, Los Alamos, 1988.

[4]A. Ferrari and P. Sala, Work. on Nucl. React. Data, Trieste 1996.

[5]M.A. Lone, Symp. On Neutr. Cross Sections 10-40 MeV, BNL- NCS-50681 (1977).

[6]H.H. Barschall, Ann. Rev. Nucl. Part. Sci. 28 (1978) 207.

[7]H.L. Ravn et al., Nucl. Instrum. Meth. B88 (1994) 441.

[8]B. Fogelberg et al., Nucl. Instrum. Meth. B70 (1992) 137

[9]V. Variale, this conference. 